\title{
Voice Alteration
}

National Cancer Institute

\section{Source}

National Cancer Institute. Voice Alteration. NCI Thesaurus. Code C61043.

Change in the sound and/or speed of the voice. Causes include laryngeal polyp, laryngitis, laryngeal carcinoma, throat carcinoma, Parkinson's disease, multiple sclerosis, stroke, hypothyroidism, oral surgery, tracheostomy, tracheal injury, and laryngeal injury. 